\title{
Epidemiological and clinical characteristics of symptomatic hereditary transthyretin amyloid polyneuropathy: a global case series
}

Márcia Waddington-Cruz ${ }^{1,8^{*}}$, Hartmut Schmidt ${ }^{2}$, Marc F. Botteman ${ }^{3}$, John A. Carter ${ }^{4} \mathbb{B}$, Michelle Stewart ${ }^{5}$, Markay Hopps ${ }^{6}$, Shari Fallet ${ }^{6}$ and Leslie Amass ${ }^{7}$

\begin{abstract}
We describe 542 cases of symptomatic hereditary transthyretin amyloid polyneuropathy (ATTR-PN) identified through a review of the literature published between 2005 and 2016. Approximately 18\% of the cases were from countries where ATTR-PN is traditionally considered to be endemic (i.e., Portugal, Japan, and Sweden). East Asia (Japan, China, Taiwan, and South Korea) contributed a sizeable combined proportion $(37.0 \%, n=200)$ with Japan $(n=92)$ and China $(n=71)$ being the primary contributors. The most common genotypes among the 65 genotypes represented in the sample were Val30Met (47.6\%), Ser77Tyr (10\%), Ala97Ser (6.5\%), and Phe64Leu (4.4\%). Cases with genotypes other than the aforementioned four had the lowest ages at onset (mean 49.2 [standard deviation $\{S D\} 21.0$; inter-quartile range $\{\mid \mathrm{QR}\} 14.7]$ ) and diagnosis (mean 53.4 [SD 21.0; IQR 14.7]). Conversely, Phe64Leu mean age of onset was 67.5 (SD 8.8; IQR 5.2) and mean age of diagnosis was 71.3 (SD 8.8; IQR 5.4). The prevalence of upper and lower limb involvement at the time of diagnosis (67 and $41 \%$ ) observed across all cases is consistent with the typical presentation of ATTR-PN. Other notable findings at the time of diagnosis included a high rate of impotence among the Ala97Ser cases versus all others (67\% vs. $21 \%$ ) and a high rate of non-motor visual symptoms (i.e., visual opacities and glaucoma) in the Ser77Tyr cases versus all others (93\% vs. 16\%). Though comparisons were made descriptively and were hindered by inconsistency of reporting across the cases, these findings support the notion that ATTR-PN is a more phenotypically and geographically variable disease than is typically considered.
\end{abstract}

Keywords: Transthyretin amyloidosis, Peripheral neuropathy, Case series, Rare disease

\section{Introduction}

Transthyretin amyloid polyneuropathy (ATTR-PN) is a rare genetic disease considered to be endemic to Portugal, Sweden, and foci in Japan [1]. Its global prevalence is traditionally and somewhat anecdotally estimated as 5000 to 10,000 [2, 3], but a recently published analysis reported that global prevalence may be as high as 38,000 persons [4]. In ATTR-PN, misfolded amyloid deposits accumulate on the peripheral nerves

\footnotetext{
* Correspondence: mwaddingtoncruz@gmail.com

${ }^{1}$ Federal University of Rio de Janeiro, Rio de Janeiro, Brazil

${ }^{8}$ Hospital Universitário Clementino Fraga Filho (HUCFF), Universidade Federal do Rio de Janeiro (UFRJ), Rio de Janeiro, Brazil

Full list of author information is available at the end of the article
}

and within major organs leading to progressive debilitating sensorimotor polyneuropathy and autonomic dysfunction [5]. This may be manifested by motor impairment, muscle weakness and wasting, and multiple organ failure, but the disease is phenotypically heterogeneous [6]. In nearly all cases ATTR-PN will progress and lead to loss of bodily function, diminished quality of life, and death within approximately $10-15$ years after onset, often due to cardiac complications [7-11].

Much of what is known about ATTR-PN has been gathered from the study of the most common genotype, Val30Met (i.e., substitution of valine for methionine in position 30 of the transthyretin protein), which in 1984 was the first causative mutation to be identified [12]. 
The clinical course of ATTR-PN in endemic countries where Val30Met predominates typically consists of symptom onset with sensory-motor symptoms. Some patients may also present with autonomic neuropathy with or without sensory-motor involvement. The age of onset in endemic regions such as Portugal and Brazil generally occurs in the mid-30's or 40's while in Sweden onset is much later (age 60-70 years). Similar to Swedish patients, in non-endemic countries, patients with the Val30Met mutation may experience the onset of symptoms at a later age [13]. Thus, in just this one genotype there are clinically important differences in age of onset and how ATTR-PN is expressed. Nearly 100 ATTR genotypes have been identified across approximately 40 countries [4, 13, 14]. This along with the associated phenotypic variability underscores the heterogeneity of this rare disease.

Partly due to this heterogeneity, a knowledge gap exists with regard to recognizing ATTR-PN, particularly in non-endemic countries where the prevalence of non-Val30Met genotypes is greater, which has led to delayed or under-diagnosis and ultimately to suboptimal treatment outcomes [15]. Although likely largely driven by lack of clinician experience and insufficient patient access to specialized treatment centers, the knowledge gap may also be attributable to a lack of consolidated case information in the literature. Published information is often specific to a single geography, institution, or genotype, making it difficult to gain insights into commonalities and differences. We conducted a broad review and synthesis of existing reports of ATTR-PN cases in an effort to develop a more comprehensive view of the clinical presentation of this disease with respect to its sensorimotor characteristics.

\section{Methods}

\section{Literature review}

A previously reported systematic review conducted according to modified Preferred Reporting Items for Reviews and Meta-Analyses (PRISMA) [16] guidelines was used to identify and synthesize ATTR-PN prevalence information globally [4]. The systematic review included structured searches of the peer-reviewed literature published from 2005 to 2016 (inclusive) via the following online reference databases: Embase, PubMed, SCOPUS, and Web of Science. Additionally, the proceedings of the following five conferences were reviewed: (a) First European Congress on Hereditary ATTR Amyloidosis (ATTR 2015); (b) International Society of Amyloidosis (ISA 2010, 2012, and 2014); (c) International Symposium on Familial Amyloidotic Polyneuropathy (ISFAP 2013).

These searches were conducted without regard to language or geography. While conducting the review of prevalence information, individual ATTR-PN clinical cases were identified and retained for further analysis.

\section{Case eligibility, data extraction, and analysis}

For each case identified, data for the following variables were collected and constituted the minimum threshold for retention in the database as a case: (A) confirmation of symptomatic ATTR-PN manifested by explicitly noted polyneuropathy, (B) sex, (C) mutation, and location / country. The following were also extracted where reported: age of (E) symptom onset, (F) diagnosis, (G) death, symptoms at $(\mathrm{H})$ onset, and (I) diagnosis, and (J) parent-of-origin effect (genotypically confirmed). Reported symptoms ascribed in the reports as being of a neuropathic nature were further categorized as autonomic, sensory, motor, and miscellaneous (i.e., cardiomyopathy, motor (non-visual), and weight-loss/anorexia) according to the taxonomy depicted in Fig. 1. Duplicate cases were identified by overlap of variables B-I and subsequently removed, as were cases of de novo disease subsequent to liver transplantation. Descriptive analysis of extracted case data addressed the following:

- Distribution of ATTR-PN genotypes by country of origin

- Age at disease milestones (onset, diagnosis, death) by genotype

- Symptoms reported at initial presentation by genotype

To address anticipated skewness, the mean, standard deviation (SD), and inter-quartile range (IQR) were calculated for disease milestone outcomes using only the values between the first and third quartiles of the extracted data. Time between disease milestones was calculated across only the cases with both values reported.

\section{Results}

There were 653 cases extracted from the literature initially. After applying eligibility criteria and removing duplicative reports, 111 cases from 15 reports were excluded. Seventy exclusions (63\%) were due to sex not having been reported, while $28(25 \%)$ of the cases were excluded because they were described as "asymptomatic" and/or no neuropathic symptoms were explicitly described. Seventeen genotypes were represented in the excluded cases, of which $62 \%$ were Val30Met followed by $11 \%$ Gly83Arg.

The retained sample included 542 cases contributed by 108 individual reports across 32 countries (Table 1). Approximately $18 \%$ of the cases were from countries where ATTR-PN is traditionally considered to be endemic (i.e., Portugal, Japan, and Sweden) [17]. Most cases were from Western Europe and the Asia-Pacific region, specifically East Asia. The four most common genotypes among the 65 genotypes represented in the sample were Val30Met (47.6\%), Ser77Tyr (10\%), Ala97Ser (6.5\%), and Phe64Leu (4.4\%) (Table 1, Additional file 1: Appendix A). 


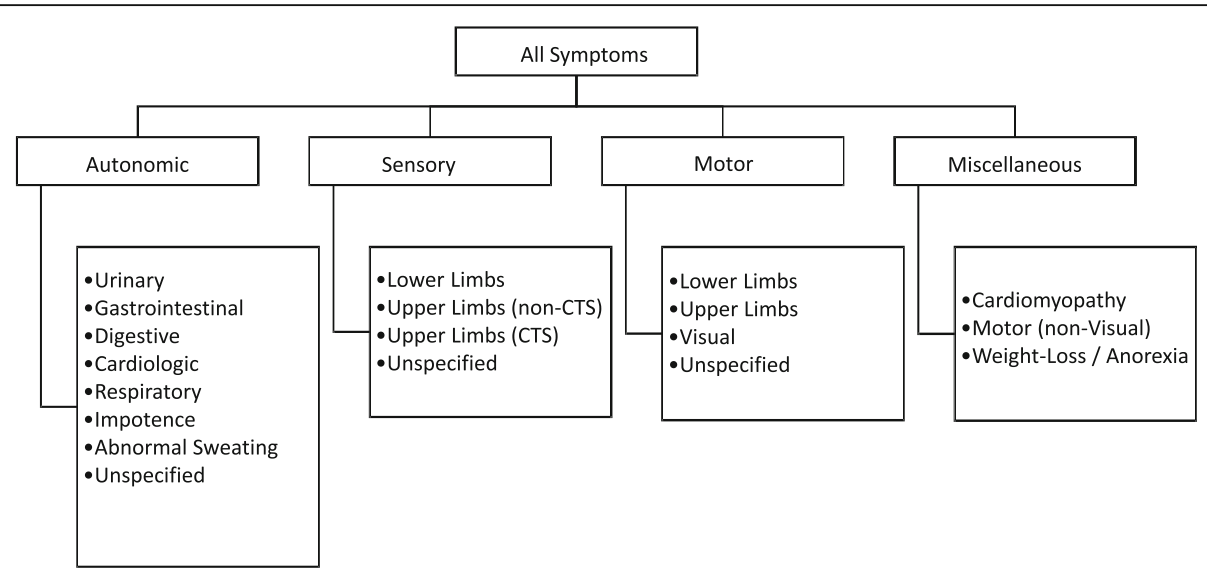

Fig. 1 This figure depicts the taxonomy of ATTR-PN symptoms extracted for analysisCTS is carpal tunnel syndrome

Val30Met was the most prevalent genotype reported in endemic countries, whereas genotypes from non-endemic countries primarily belonged to the "Other" category (i.e., those comprising $<4 \%$ of the retained cases). There was insufficient data to assess genotypically-confirmed parent-of-origin effect. Ages at onset of neuropathy, diagnosis, and death were reported for $n=394, n=276$, and $n$ $=139$ cases, respectively. Summary statistics for these milestones are listed in Table 2. It was difficult to draw meaningful inter-genotype comparisons from these data due to the heterogeneous nature of the reporting, and because assessment of time from onset or diagnosis to death was biased by right-censoring.

Referring to Table 2, cases with genotypes in the "other" category had the lowest ages at onset (Mean 49.2 [SD 21.0; IQR 14.7]) and diagnosis (Mean 53.4 [SD 21.0; IQR 14.7]). Conversely, Phe64Leu mean age of onset was 67.5 (SD 8.8; IQR 5.2) and mean age of diagnosis was 71.3 (SD 8.8; IQR 5.4). The mean age of death (uncorrected for censoring and individual case characteristics) for Ser77Tyr was the lowest among all groups (Mean 58.5 [SD 5.8; IQR 4.2]).

Table 3 lists the proportions of cases with given symptoms reported at diagnosis stratified by genotype. Eighty-seven percent of all cases reported sensory neuropathy at diagnosis [18]. Note that all cases retained for this analysis were confirmed by the reporting author(s) to be diagnosed with ATTR-PN and were explicitly described as having experienced sensory neuropathy during the course of their disease that was attributable to ATTR-PN. The methods used to establish these diagnoses were not recorded for the present review. In many reports, the cases received an initial diagnosis after presenting with prolonged gastrointestinal symptoms or abnormal cardiologic findings (e.g., arrhythmia and other cardiac autonomic abnormalities). Among the cases reporting sensory neuropathy at the time of diagnosis, more had lower limb versus upper limb involvement $(67 \%$ vs. 41\%), which is consistent with the characterization of ATTR-PN sensory neuropathy originating in the feet and later spreading to the upper limbs as the disease progresses [19]. Other notable findings at the time of diagnosis included a relatively high rate of impotence among the Ala97Ser cases versus all others $(67 \%$ vs. $21 \%)$ and a high rate of non-motor visual symptoms (i.e., visual opacities and glaucoma) in the Ser77Tyr cases versus all others (93\% vs. $16 \%$ ).

\section{Discussion}

A search of bibliographic databases and the proceedings of amyloidosis-focused clinical conferences yielded 542 unique ATTR-PN cases. Four genotypes (Val30Met, Ser77Tyr, Ala97Ser, and Phe64Leu) comprised $70 \%$ of the total case sample, while 65 genotypes were identified overall. France was the largest single locus of cases $(17.9 \%, n=97)$ among 32 countries represented; however, East Asia (Japan, China, Taiwan, and South Korea) contributed a sizeable combined proportion $(37.0 \%, n=200)$ with Japan $(n=92)$ and China $(n=71)$ being the primary contributors. The remaining 245 cases originated mostly from Western Europe: particularly Italy $(n=58)$ and Germany $(n=26)$.

There were notable findings with regard to the timing of key disease milestones (i.e., onset, diagnosis, and death). For example, the mean age of symptom onset across ATTR-PN cases included here was $61.5( \pm 11.5)$ years, whereas traditionally, disease onset has been reported to occur by age $50[15,20,21]$. Seemingly dissonant findings are explained by examining the characteristics of cases in previous reports versus the present one. Published estimates for the timing of disease milestones are heavily influenced by patients with Portuguese-type Val30Met disease in endemic countries, which is the most prevalent form and for which onset is typically at $30-40$ years old [15], and generally onset is 
Table 1 Global Distribution of Reviewed ATTR-PN Cases, n (\%)

\begin{tabular}{|c|c|c|c|c|c|c|}
\hline Country & Country Total & Ala97Ser & Phe64Leu & Ser77Tyr & Val30Met & Other \\
\hline France & 97 (17.9\%) & $0(0 \%)$ & $0(0 \%)$ & 33 (34\%) & $47(48.5 \%)$ & $17(17.5 \%)$ \\
\hline Japan & 92 (17\%) & $0(0 \%)$ & $0(0 \%)$ & $0(0 \%)$ & 76 (82.6\%) & 16 (17.4\%) \\
\hline China & $71(13.1 \%)$ & $2(2.8 \%)$ & $0(0 \%)$ & $12(16.9 \%)$ & $23(32.4 \%)$ & 34 (47.9\%) \\
\hline Italy & $58(10.7 \%)$ & $0(0 \%)$ & $24(41.4 \%)$ & $0(0 \%)$ & $21(36.2 \%)$ & 13 (22.4\%) \\
\hline Taiwan & 35 (6.5\%) & 33 (94.3\%) & $0(0 \%)$ & $1(2.9 \%)$ & $0(0 \%)$ & 1 (2.9\%) \\
\hline Germany & $26(4.8 \%)$ & $0(0 \%)$ & $0(0 \%)$ & $0(0 \%)$ & $13(50 \%)$ & $13(50 \%)$ \\
\hline Spain & 19 (3.5\%) & $0(0 \%)$ & $0(0 \%)$ & $0(0 \%)$ & $8(42.1 \%)$ & 11 (57.9\%) \\
\hline Portugal & $18(3.3 \%)$ & $0(0 \%)$ & $0(0 \%)$ & $0(0 \%)$ & 16 (88.9\%) & $2(11.1 \%)$ \\
\hline Greece & 17 (3.1\%) & $0(0 \%)$ & $0(0 \%)$ & $0(0 \%)$ & 17 (100\%) & $0(0 \%)$ \\
\hline Israel & 17 (3.1\%) & $0(0 \%)$ & $0(0 \%)$ & $8(47.1 \%)$ & $4(23.5 \%)$ & $5(29.4 \%)$ \\
\hline Sweden & $14(2.6 \%)$ & $0(0 \%)$ & $0(0 \%)$ & $0(0 \%)$ & $5(35.7 \%)$ & 9 (64.3\%) \\
\hline Ireland & $12(2.2 \%)$ & $0(0 \%)$ & $0(0 \%)$ & $0(0 \%)$ & $0(0 \%)$ & 12 (100\%) \\
\hline Argentina & $11(2 \%)$ & $0(0 \%)$ & $0(0 \%)$ & $0(0 \%)$ & $8(72.7 \%)$ & $3(27.3 \%)$ \\
\hline Turkey & $11(2 \%)$ & $0(0 \%)$ & $0(0 \%)$ & $0(0 \%)$ & $4(36.4 \%)$ & 7 (63.6\%) \\
\hline Brazil & $8(1.5 \%)$ & $0(0 \%)$ & $0(0 \%)$ & $0(0 \%)$ & $8(100 \%)$ & $0(0 \%)$ \\
\hline United States & $6(1.1 \%)$ & $0(0 \%)$ & $0(0 \%)$ & $0(0 \%)$ & 1 (16.7\%) & $5(83.3 \%)$ \\
\hline Australia & $3(0.6 \%)$ & $0(0 \%)$ & $0(0 \%)$ & $0(0 \%)$ & $0(0 \%)$ & $3(100 \%)$ \\
\hline Belgium & $3(0.6 \%)$ & $0(0 \%)$ & $0(0 \%)$ & $0(0 \%)$ & $3(100 \%)$ & $0(0 \%)$ \\
\hline Finland & $3(0.6 \%)$ & $0(0 \%)$ & $0(0 \%)$ & $0(0 \%)$ & $0(0 \%)$ & $3(100 \%)$ \\
\hline Poland & $3(0.6 \%)$ & $0(0 \%)$ & $0(0 \%)$ & $0(0 \%)$ & $0(0 \%)$ & $3(100 \%)$ \\
\hline Romania & $3(0.6 \%)$ & $0(0 \%)$ & $0(0 \%)$ & $0(0 \%)$ & $0(0 \%)$ & $3(100 \%)$ \\
\hline Korea (South) & $2(0.4 \%)$ & $0(0 \%)$ & $0(0 \%)$ & $0(0 \%)$ & $0(0 \%)$ & $2(100 \%)$ \\
\hline Russia & $2(0.4 \%)$ & $0(0 \%)$ & $0(0 \%)$ & $0(0 \%)$ & $0(0 \%)$ & $2(100 \%)$ \\
\hline Slovenia & $2(0.4 \%)$ & $0(0 \%)$ & $0(0 \%)$ & $0(0 \%)$ & $0(0 \%)$ & $2(100 \%)$ \\
\hline Switzerland & $2(0.4 \%)$ & $0(0 \%)$ & $0(0 \%)$ & $0(0 \%)$ & $1(50 \%)$ & $1(50 \%)$ \\
\hline Czech Republic & $1(0.2 \%)$ & $0(0 \%)$ & $0(0 \%)$ & $0(0 \%)$ & $0(0 \%)$ & $1(100 \%)$ \\
\hline Denmark & $1(0.2 \%)$ & $0(0 \%)$ & $0(0 \%)$ & $0(0 \%)$ & $1(100 \%)$ & $0(0 \%)$ \\
\hline Holland & $1(0.2 \%)$ & $0(0 \%)$ & $0(0 \%)$ & $0(0 \%)$ & $1(100 \%)$ & $0(0 \%)$ \\
\hline India & $1(0.2 \%)$ & $0(0 \%)$ & $0(0 \%)$ & $0(0 \%)$ & $0(0 \%)$ & 1 (100\%) \\
\hline Malaysia & $1(0.2 \%)$ & $0(0 \%)$ & $0(0 \%)$ & $0(0 \%)$ & $0(0 \%)$ & $1(100 \%)$ \\
\hline Norway & $1(0.2 \%)$ & $0(0 \%)$ & $0(0 \%)$ & $0(0 \%)$ & $1(100 \%)$ & $0(0 \%)$ \\
\hline United Kingdom & $1(0.2 \%)$ & $0(0 \%)$ & $0(0 \%)$ & $0(0 \%)$ & $0(0 \%)$ & 1 (100\%) \\
\hline Genotype Total & $542(100 \%)$ & 35 (6.5\%) & $24(4.4 \%)$ & $54(10 \%)$ & $258(47.6 \%)$ & 171 (31.5\%) \\
\hline
\end{tabular}

Specific genotypes shown are those with $\geq 4 \%$ representation among the included cases. Genotypes with $<4 \%$ representation are listed as "Other". Refer to Additional file 1: Appendix A for a list of genotypes included in the "Other" category

earlier in endemic versus non-endemic countries (except in Sweden where it is typically late-onset). Furthermore, previous case series have reported that age of onset is later for non-Val30Met ATTR-PN [21, 22]. Thus, the later onset reported here appears consistent with the aforementioned trends given that cases were predominantly from non-endemic countries and non-Val30Met (Table 2).

The relatively higher onset age reported here might also reflect changing trends in disease characteristics at presentation that have been influenced by better disease awareness and population factors such as decreasing fertility rates. In their recently published epidemiological assessment of ATTR-PN in Portugal, Ines et al. (2018) implicated these same factors as likely reasons for a higher incident age [23]. The authors noted that the ratio of late-onset to early-onset incident cases nearly doubled from 1:4 (22.4\%) to 2:4 (44.4\%) between 2010 and 2016. They ascribed this to the upward influence on late-onset diagnoses generated by better late-onset disease recognition, and the downward influence on early-onset cases generated by a $50 \%$ decline in the 
Table 2 Characteristics of 542 ATTR-PN cases

\begin{tabular}{|c|c|c|c|c|c|c|}
\hline & All & Ala97Ser & Phe64Leu & Ser77Tyr & Val30Met & Other \\
\hline N & 542 & 35 & 24 & 54 & 258 & 171 \\
\hline$\%$ of Sample & $(100 \%)$ & $(6.5 \%)$ & $(4.4 \%)$ & $(10.0 \%)$ & $(47.6 \%)$ & $(31.5 \%)$ \\
\hline$\%$ from Endemic ${ }^{a}$ & $17.9 \%$ & $0.0 \%$ & $0.0 \%$ & $0.0 \%$ & $37.6 \%$ & $15.8 \%$ \\
\hline$\%$ Male & $68.6 \%$ & $85.7 \%$ & $79.2 \%$ & $74.1 \%$ & $69.0 \%$ & $61.4 \%$ \\
\hline \multicolumn{7}{|c|}{ Disease Milestones Mean (SD, IQR) [years] } \\
\hline Onset & $61.5(11.5 ; 8.4)$ & $58.5(8.0 ; 6.4)$ & $67.5(8.8 ; 5.2)$ & $51.6(12.0 ; 8.2)$ & $64.0(12.0 ; 8.1)$ & $49.2(21.0 ; 14.7)$ \\
\hline Diagnosis & $64.2(13.6 ; 9.6)$ & $58(2.0 ; 2.2)$ & $71.3(8.8 ; 5.4)$ & $57.7(9.5 ; 6.4)$ & $68.1(8.1 ; 6.7)$ & $53.4(21.0 ; 14.7)$ \\
\hline Death & $66.3(14.0 ; 9.9)$ & - & - & $58.5(5.8 ; 4.2)$ & $71.0(9.5 ; 7.4)$ & $65.7(15.3 ; 10.1)$ \\
\hline Onset to Diagnosis & $2.9(3.2 ; 2.1)$ & $8.6(3.0 ; 2.1)$ & $3.8(2.0 ; 1.8)$ & $2.3(2.7 ; 1.5)$ & $3.0(3.4 ; 2.4)$ & $2.7(3.0 ; 2.2)$ \\
\hline Diagnosis to Death & $1.9(2.0 ; 1.4)$ & - & - & $1.1(1.1 ; 0.6)$ & $2.1(1.9 ; 1.3)$ & $2.1(1.8 ; 1.4)$ \\
\hline Onset to Death & $5.0(3.0 ; 2.4)$ & - & - & $3.9(2.8 ; 2.1)$ & $5.9(4.0 ; 2.8)$ & $5.4(2.8 ; 1.8)$ \\
\hline
\end{tabular}

Specific genotypes shown are those with $\geq 4 \%$ representation among the included cases. Genotypes with $<4 \%$ representation are listed as "Other". Refer to Additional file 1: Appendix A for a list of genotypes included in the "Other" category. Refer to Additional file 1: Appendix B for data used to generate disease milestone outcomes. IQR is inter-quartile range. SD is standard deviation

a Japan, Portugal, and Sweden were categorized as endemic countries

Table 3 Clinical Characteristics at Presentation

\begin{tabular}{|c|c|c|c|c|c|c|}
\hline Feature & All & Ala97Ser & Phe64Leu & Ser77Tyr & Val30Met & Other \\
\hline Any Reported & $374(69 \%)$ & $35(9 \%)$ & $22(6 \%)$ & 48 (13\%) & $141(38 \%)$ & $128(34 \%)$ \\
\hline Autonomic & 199 (53\%) & 27 (77\%) & $18(82 \%)$ & $5(10 \%)$ & 74 (52\%) & 75 (59\%) \\
\hline Unspecified & 47 & 0 & 7 & 1 & 22 & 17 \\
\hline Urinary & 36 & 7 & 3 & 0 & 16 & 10 \\
\hline Gastrointestinal & 114 & 22 & 6 & 3 & 39 & 44 \\
\hline Cardio & 96 & 15 & 7 & 1 & 34 & 39 \\
\hline Respiratory & 3 & 0 & 0 & 0 & 0 & 3 \\
\hline Impotence & 51 & 18 & 5 & 1 & 13 & 14 \\
\hline Sweat & 20 & 7 & 3 & 1 & 4 & 5 \\
\hline Sensory & $326(87 \%)$ & 25 (71\%) & 22 (100\%) & 45 (94\%) & 127 (90\%) & 107 (84\%) \\
\hline Unspecified & 76 & 21 & 5 & 0 & 25 & 25 \\
\hline Lower Limbs & 219 & 2 & 15 & 37 & 100 & 65 \\
\hline Upper Limbs & 133 & 2 & 10 & 35 & 48 & 38 \\
\hline Carpal Tunnel & 42 & 4 & 4 & 0 & 7 & 27 \\
\hline Motor & 215 (57\%) & 10 (29\%) & $15(68 \%)$ & 32 (67\%) & 79 (56\%) & 79 (62\%) \\
\hline Unspecified & 67 & 9 & 4 & 0 & 33 & 21 \\
\hline Lower Limbs & 138 & 0 & 11 & 28 & 46 & 53 \\
\hline Upper Limbs & 83 & 1 & 10 & 10 & 33 & 29 \\
\hline Other & 1 & 0 & 0 & 0 & 0 & 1 \\
\hline Miscellaneous & $155(41 \%)$ & $4(11 \%)$ & 17 (77\%) & $14(29 \%)$ & $39(28 \%)$ & 81 (63\%) \\
\hline Cardiomyopathy & 93 & 4 & 15 & 2 & 28 & 44 \\
\hline Visual (Non-Motor) & 49 & 0 & 2 & 13 & 9 & 25 \\
\hline Weight Loss & 35 & 0 & 2 & 2 & 9 & 22 \\
\hline
\end{tabular}


national fertility rate over the past 40 years. This trend will likely accelerate as genetic counseling and medical-assisted reproductive methods instituted over the past three decades begin to have a more demonstrable effect in reducing carrier prevalence [23].

After recognizing that the cases included here were atypical in the sense that they were generally later-onset and non-Val30Met, it was also important to examine how this might be related to the observed time between onset and death. The reported mean time from symptom onset to death in persons with ATTR-PN is 10-15 years [14, 24]. In the present review this value was 5 years. This descriptive assessment did not adjust for censoring or case characteristics, and the potential biasing effects cannot be overlooked. However, this discrepancy between average values in the literature and our findings may be somewhat attributable to geographic origin and genotype. Our sample was largely atypical and sporadic. As has been noted previously, sporadic cases may not receive adequate treatment as early as more typical cases (i.e., those presenting in endemic areas with Val30Met disease). Variable clinical features combined with limited physician awareness and insufficient diagnostic capabilities in non-endemic areas may have resulted in delayed diagnosis and treatment, which would expedite disease progression and death [25]. With regard to our analysis of symptoms at onset and diagnosis, we observed a typical progression [26] overall in that sensory disturbances generally originated in the distal lower extremities and spread proximally. The contention that our sample represents a more sporadic and later-onset population in which diagnosis was delayed is supported by observed relatively high rates of motor and autonomic dysfunction, both hallmarks of progressed disease [26, 27]. This coupled with the observed average age of symptom onset and signs of progressed disease at diagnosis provide further support for the conclusion that these cases were generally late-onset and received delayed diagnoses. It is also notable that many cases were initially diagnosed incorrectly with chronic inflammatory demyelinating polyneuropathy, which is consistent with previously reported cases described as sporadic and late-onset [20].

While this case series provides useful information regarding the genotypic, phenotypic, and geographic variability of ATTR-PN, our descriptive analysis was limited by inconsistency among the individual case reports. For example, in $13 \%$ of cases there was no description of sensory neuropathy despite all cases having reportedly been diagnosed with symptomatic ATTR-PN for which sensory neuropathy is the most common initial symptom. Furthermore, the sample size was not sufficient to draw statistically robust comparisons among genotypes for the timing of disease milestones and the composition of symptoms at onset/diagnosis. It is also possible that selective reporting of novel mutations and sporadic cases may have biased the results, and noted differences in symptoms might reflect differences in data collection rather than disease presentation. Lastly, this review was limited to the sensorimotor characteristics of ATTR-PN, but this is not a complete clinical picture of the disease, particularly in countries like the United States and United Kingdom where cardiac involvement - namely heart failure with preserved ejection fraction - is the predominant presenting characteristic for the hereditary form of the disease.

Despite some limitations, these case reports are an important resource for this rare, progressive, and generally fatal disease. Non-endemic regions for example have few patients, but disproportionately many of them are sporadic cases for which a positive family history of ATTR-PN - typically among the most apparent risk factors [27] - is either lacking or not evaluable to facilitate timely diagnosis. This point cannot be overstated because without adequate information, the pattern of sensory-motor and autonomic neuropathy in patients with early ATTR-PN who would benefit most from treatment may be indistinguishable from more common diagnoses [27]. This report is also relevant in endemic areas because it emphasizes that their true ATTR-PN populations likely extend beyond historically predominant genotypes and phenotypes. Overall, knowledge of ATTR-PN appears largely derived from endemic areas and persons with early-onset Val30Met disease because the disease is exceedingly rare otherwise. It is hoped that comprehensive case series such as this will help broaden the understanding of ATTR-PN - providing insight into non-endemic areas and less common genotypes - so that afflicted persons can receive prompt, accurate diagnosis and begin treatment when it will be most effective.

\section{Additional file}

Additional file 1: Appendix A. Genotypes Included in the "Other" Category. Appendix B. Summary Statistics for Disease Milestone Outcomes without Outliers. (DOCX $20 \mathrm{~kb}$ )

\section{Abbreviations}

ATTR-PN: Transthyretin amyloid polyneuropathy; IQR: Inter-quartile range; PRISMA: Preferred Reporting Items for Systematic Reviews and MetaAnalyses; SD: Standard deviation

\section{Acknowledgements}

The authors wish to acknowledge and thank Dr. Avijeet S Chopra, PhD formerly of Pharmerit International - for his editorial assistance in drafting this manuscript.

\section{Funding}

This research was supported by Pfizer Inc.

Availability of data and materials

The datasets used and/or analyzed during the current study are available from the corresponding author on reasonable request. 


\section{Authors' contributions}

All authors contributed equally to the development, execution, and report of this work. All authors read and approved the final manuscript.

\section{Authors' information}

MWC is Chief of the Neuromuscular Diseases Unit at the University Hospital, Federal University of Rio, Brazil; and has been overseeing the Familial Amyloid Polyneuropathy patient care clinic (CEPARM) at the hospital for over 20 years. MWC also serves as the chair of the Transthyretin Amyloidosis Outcomes Survey (THAOS).

\section{Ethics approval and consent to participate} Not Applicable.

\section{Consent for publication}

Not Applicable.

\section{Competing interests}

MS, MH, and LA are employees of Pfizer Inc. SF was an employee of Pfizer Inc. at the time this research was conducted. MFB is an employee of Pharmerit International, which received funding from Pfizer Inc. for study design, execution, analysis, and manuscript development. ASC was an employee of Pharmerit International at the time this research was conducted. JAC is an independent scientific consultant funded by Pharmerit International. HS and MWC were investigators for the study and were not financially compensated for collaborative efforts on publication-related activities. HS received support from FoldRx Pharmaceuticals, which was acquired by Pfizer Inc. in October 2010, as a clinical investigator. MWC received support from FoldRx Pharmaceuticals, which was acquired by Pfizer Inc. in October 2010, as a clinical investigator; has served on the scientific advisory board of Pfizer Inc.; received funding from Pfizer Inc. for scientific meeting expenses (travel, accommodations, and registration); and received research support from the National Institutes of Health. She currently serves on the THAOS (natural history disease registry) scientific advisory board.

\section{Publisher's Note}

Springer Nature remains neutral with regard to jurisdictional claims in published maps and institutional affiliations.

\section{Author details}

${ }^{1}$ Federal University of Rio de Janeiro, Rio de Janeiro, Brazil. ${ }^{2}$ Muenster University Hospital, Muenster, Germany. ${ }^{3}$ Pharmerit International, Bethesda, MD, USA. ${ }^{4}$ BluePoint, LLC, Chicago, IL, USA. ${ }^{5}$ Pfizer Inc., Groton, CT, USA. ${ }^{6}$ Pfizer Inc., New York, NY, USA. ${ }^{7}$ Pfizer Inc., Collegeville, PA, USA. ${ }^{8}$ Hospital Universitário Clementino Fraga Filho (HUCFF), Universidade Federal do Rio de Janeiro (UFRJ), Rio de Janeiro, Brazil.

\section{Received: 30 March 2018 Accepted: 8 January 2019}

Published online: 08 February 2019

\section{References}

1. Waddington-Cruz M, Schmidt H, Botteman MF, et al. Epidemiological and clinical characteristics of persons with transthyretin familial amyloid polyneuropathy: A global synthesis of 532 cases. In: International Society of Amyloidosis - XV International Symposium on Amyloidosis (ISA); 2016.

2. Plante-Bordeneuve $\mathrm{V}$. Update in the diagnosis and management of transthyretin familial amyloid polyneuropathy. J Neurol. 2014;261(6):1227-33. https://doi.org/10.1007/s00415-014-7373-0.

3. Misu Kl, Hattori N, Nagamatsu M, et al. Late-onset familial amyloid polyneuropathy type I (transthyretin Met30-associated familial amyloid polyneuropathy) unrelated to endemic focus in Japan. Clinicopathological and genetic features. Brain. 1999;122(1):1951-62.

4. Schmidt HH, Waddington-Cruz M, Botteman MF, et al. Estimating the global prevalence of transthyretin familial amyloid polyneuropathy. Muscle Nerve. 2018;57(5):829-37. https://doi.org/10.1002/mus.26034.

5. Yamashita T, Ando Y, Okamoto S, et al. Long-term survival after liver transplantation in patients with familial amyloid polyneuropathy. Neurology. 2012;78(9):637-43. https://doi.org/10.1212/WNL.0b013e318248df18.

6. Ikeda S. Transthyretin Val30Met familial amyloid polyneuropathy: a considerably different clinical picture and natural course in endemic and non-endemic areas. J Neurol Neurosurg Psychiatry. 2012;83(2):121 http://jnnp.bmj.com/content/83/2/121.abstract.

7. Reinés JB, Vera TR, Martín MU, et al. Epidemiology of transthyretinassociated familial amyloid polyneuropathy in the Majorcan area: son Llàtzer hospital descriptive study. Orphanet J Rare Dis. 2014;9:29. https://doi.org/10. 1186/1750-1172-9-29.

8. Coelho T, Maia LF, Martins da Silva A, et al. Tafamidis for transthyretin familial amyloid polyneuropathy: a randomized, controlled trial. Neurology. 2012;79(8):785-92. https://doi.org/10.1212/WNL.0b013e3182661eb1.

9. Bekircan-Kurt C, Gunes N, Yilmaz A, Erdem-Ozdamar S, Tan E. Three Turkish families with different transthyretin mutations. Neuromuscul Disord. 2015;25: 686-92. https://doi.org/10.1016/j.nmd.2015.05.010.

10. Bulawa C, Connelly S, Devit M, et al. Tafamidis, a potent and selective transthyretin kinetic stabilizer that inhibits the amyloid cascade. Proc Natl Acad Sci U S A. 2012;109(24):9629-34. https://doi.org/10.1073/pnas.1121005109.

11. Rudolph T, Kurz MW, Farbu E. Late-onset familial amyloid polyneuropathy (FAP) Val30Met without family history. Clin Med Res. 2008;6(2):80-2. https:// doi.org/10.3121/cmr.2008.794.

12. Saraiva MJ, Birken S, Costa PP, Goodman DS. Family studies of the genetic abnormality in transthyretin (prealbumin) in Portuguese patients with familial amyloidotic polyneuropathy. Ann N Y Acad Sci. 1984;435:86-100.

13. Cruz MW. Regional differences and similarities of familial amyloidotic polyneuropathy (FAP) presentation in Brazil. Amyloid. 2012;19(sup1):65-7. https://doi.org/10.3109/13506129.2012.673183.

14. Araki S, Ando Y. Transthyretin-related familial amyloidotic polyneuropathyProgress in Kumamoto, Japan (1967-2010). Proc Jpn Acad Ser B Phys Biol Sci. 2010;86(7):694-706.

15. Parman $Y$, Adams $D$, Obici $L$, et al. Sixty years of transthyretin familial amyloid polyneuropathy (TTR-FAP) in Europe: where are we now? A European network approach to defining the epidemiology and management patterns for TTR-FAP. Curr Opin Neurol. 2016;29(Suppl 1):S3S13. https://doi.org/10.1097/WCO.0000000000000288.

16. Moher D, Liberati A, Tetzlaff J, Altman DG. Preferred reporting items for systematic reviews and meta-analyses: the PRISMA statement. Int I Surg. 2010;8(5):336-41. https://doi.org/10.1016/j.ijsu.2010.02.007.

17. Hellman U, Alarcon F, Lundgren H-E, Suhr OB, Bonaiti-Pellie C, PlanteBordeneuve $\mathrm{V}$. Heterogeneity of penetrance in familial amyloid polyneuropathy, ATTR Val30Met, in the Swedish population. Amyloid Int J Exp Clin Investig Off J Int Soc Amyloidosis. 2008;15(3):181-6. https://doi.org/ 10.1080/13506120802193720

18. Koike H, Misu K, Sugiura M, et al. Pathology of early- vs late-onset TTR Met30 familial amyloid polyneuropathy. Neurology. 2004;63(1):129-38.

19. Carr AS, Pelayo-Negro AL, Evans MR, et al. A study of the neuropathy associated with transthyretin amyloidosis (ATTR) in the UK. J Neurol Neurosurg Psychiatry. 2016;87(6):620-7. https:/doi.org/10.1136/jnnp-2015-310907.

20. Planté-Bordeneuve V, Ferreira A, Lalu T, et al. Diagnostic pitfalls in sporadic transthyretin familial amyloid polyneuropathy (TTR-FAP). Neurology. 2007; 69(7):693-8 http://n.neurology.org/content/69/7/693.abstract.

21. Mazzeo A, Russo M, Di Bella G, et al. Transthyretin-related familial amyloid polyneuropathy (TTR-FAP): a single-center experience in Sicily, an Italian endemic area. J Neuromuscul Dis. 2015;2(s2):S39-48. https://doi.org/10. 3233/JND-150091.

22. Meng L-C, Lyu H, Zhang W, Liu J, Wang Z-X, Yuan Y. Hereditary transthyretin amyloidosis in eight Chinese families. Chin Med J. 2015; 128(21):2902-5. https://doi.org/10.4103/0366-6999.168048.

23. Inês $\mathrm{M}$, Coelho T, Conceição I, Duarte-Ramos F, de Carvalho M, Costa J. Epidemiology of transthyretin familial amyloid polyneuropathy in Portugal: a Nationwide study. Neuroepidemiology. 2018;51(3-4):177-82. https://doi. org/10.1159/000490553.

24. Plante-Bordeneuve $\mathrm{V}$. Transthyretin familial amyloid polyneuropathy: an update. J Neurol. 2018;265(4):976-83. https://doi.org/10.1007/s00415-017-8708-4.

25. Adams D, Suhr OB, Hund E, et al. First European consensus for diagnosis, management, and treatment of transthyretin familial amyloid polyneuropathy. Curr Opin Neurol. 2016;29(Suppl 1):S14-26. https://doi.org/ 10.1097/WCO.0000000000000289.

26. Ando Y, Coelho T, Berk JL, et al. Guideline of transthyretin-related hereditary amyloidosis for clinicians. Orphanet J Rare Dis. 2013;8:31. https://doi.org/10. 1186/1750-1172-8-31.

27. Conceicao I, Gonzalez-Duarte A, Obici L, et al. "Red-flag" symptom clusters in transthyretin familial amyloid polyneuropathy. J Peripher Nerv Syst. 2016; 21(1):5-9. https://doi.org/10.1111/jns.12153. 